\title{
DIAGNÓSTICOS DE ENFERMAGEM: ATRIBUIÇÃO FEITA POR GRADUANDOS DE ENFERMAGEM A PACIENTES INTERNADOS COM ALTERAÇÕES NEUROLÓGICAS
}

\author{
Maria Lúcia do Carmo Cruz Robazzi* \\ Emília Campos de Carvalho** \\ Maria Manuela Rino Mendes* \\ Eugênia Velludo Veiga*
}

ROBAZZI, M.L.do C.C.; CARVALHO, E.C.de; MENDES, M.M.R.; VEIGA, E.V. Diagnósticos de enfermagem: atribuição feita por graduandos de enfermagem a pacientes internados com alterações neurológicas. Rev.latinoam.enfermagem, Ribeirão Preto, v. 6, n. 2, p. 37-46, abril 1998.

Identificou-se categorias diagnósticas e padrões de respostas humanas em pacientes neurológicos, assistidos por alunos de graduação, que lhes atribuíram diagnósticos de enfermagem segundo a Taxonomia 1 da NANDA. A maioria dos diagnósticos referem-se aos padrões Trocar, Mover e Comunicar e a minoria aos padrões Escolher, Perceber e Sentir. A não atribuição de outros diagnósticos possivelmente ocorreu pela menor ênfase dada aos mesmos e inexperiência dos alunos. A intencionalidade dos docentes em desenvolver nos estudantes habilidades de análise e sintese, tem-se mostrado uma experiência positiva.

UNITERMOS: diagnósticos de enfermagem em pacientes neurológicos, ensino de diagnósticos de enfermagem, processo de enfermagem

\section{INTRODUÇÃO}

Há aproximadamente 20 anos, os docentes responsáveis pelo ensino da disciplina Enfermagem Médica, da Escola de Enfermagem de Ribeirão Preto da Universidade de São Paulo (EERP-USP), vêm procurando ministrar os conteúdos teórico-práticos desta disciplina, utilizando Processo de Enfermagem. A partir de 1992, encontram-se empenhados em ensinar uma metodologia, composta das fases de: coleta de dados, elaboração de Diagnósticos de Enfermagem-DE, (segundo a Taxonomia I preconizada pela North American Nursing Diagnosis Association - NANDA, divulgada no país por FARIAS et al., 1990), planejamento da assistência, implementação e avaliação da mesma.

Esta escolha aconteceu em decorrência de estarem convictos de que, neste limiar de século, era necessário a Enfermagem procurar caminhar para construir conhecimentos fundamentais, articulados e independentes dos saberes apropriados de outras profissões que, em conjunto, constroem o saber da área da saúde. Para dimensionar um corpo próprio de conhecimentos da Enfermagem que possibilite o saber cuidar, o grupo de docentes supõe que esta condição seja mais facilmente alcançada, através do ensino, aos futuros enfermeiros, da habilidade para diagnosticar, o que vem sendo, atualmente, detalhado no Processo de Enfermagem.

Evidentemente só se consegue atribuir diagnósticos a um paciente, ou seja, julgar profissional e clinicamente os problemas ou alterações apresentadas por ele, se além da inteiração alcançada, a partir do relacionamento estabelecido entre ambos - profissional e enfermo, existir global conhecimento sobre o mesmo, o que pressupõe, inclusive, o entendimento de sua fisiopatologia. A obtenção deste e de outros conhecimentos, nas esferas psicossociais/espirituais, que objetiva alcançar uma forma especializada do "cuidar", pode ser facilitada por meio da utilização de metodologia assistencial, que possibilite elaborar diagnósticos.

A princípio, utilizar DE significou rejeição por parte dos graduandos de enfermagem, que encontraram apoio em suas queixas, pelo fato de somente uma das disciplinas do elenco do curso, abordar tal conteúdo, ou mesmo pela visão até então tradicional de "enxergar" a ação do enfermeiro centrada na prescrição médica.

\footnotetext{
* Professores Doutores. Docentes da Disciplina Enfermagem Médica da Escola de Enfermagem de Ribeirão Preto da Universidade de São Paulo

** Professor Titular. Docente da Disciplina Enfermagem Médica da Escola de Enfermagem de Ribeirão Preto da Universidade de São Paulo
} 
Também os enfermeiros assistenciais comentavam com os docentes da disciplina em questão, a sua incompreensão e rejeição diante da modificação introduzida pela nova sistemática. Este tipo de comportamento é documentado por RODRIGUES \& LINS (1990), para quem, "infelizmente, muitas enfermeiras continuam a definir a enfermagem unicamente em termos de atendimentos e de procedimentos técnicos baseados na tradição e não no conhecimento, compreensão e adequação obtidos através da pesquisa. Para muitos enfermeiros, só é relevante a competência, esmero e padronização rígida das técnicas".

Esperando desenvolver, na Disciplina Enfermagem Médica, procedimentos técnicos não contemplados, em parte, em disciplinas anteriores que a antecedem no quadro do Currículo de Graduação, onde assistem os pacientes com graus de complexidade menores, os alunos relutavam em efetuar as atribuições diagnósticas, pois queixavam-se de que necessitavam estudar muito mais sobre situações aparentemente comuns, que aconteciam no cotidiano dos estágios, antes de efetivamente realizarem intervenções aos pacientes. Como era-lhes exigido primeiramente conhecer em profundidade, em etapa anterior a intervir, ou seja, a intervenção dava-se a partir deste conhecimento, as suas avaliações acerca deste novo processo eram, em geral, negativas, pois, muitas vezes deixavam de executar tarefas e técnicas em detrimento da necessidade de obter conhecimentos, de refletir, analisar e discutir acerca do porque realizá-las.

Sabe-se que as habilidades técnicas eram e ainda vem sendo exigidas pelo mercado de trabalho na área da saúde. Ao prestarem concursos de admissões em hospitais, as exigências, em geral, apresentadas pelo sistema de saúde, ainda priorizam que os enfermeiros possuam, tecnicidades, habilidades manuais e não necessariamente, conhecimentos. Este pensamento parece contar com o apoio de grande parte do empresariado da área que, possivelmente, ainda deseja contratar, enfermeiros cumpridores de tarefas, em geral alienados, dóceis e portadores de habilidades, divisores de atividades com a sua equipe e não indivíduos que tenham conhecimentos, que saibam argumentar, discutir e analisar os problemas e "ver" o paciente de modo global.

Há o entendimento por parte dos autores deste texto, que o desenvolvimento de habilidades técnicas nos enfermeiros não deve excluir a existência de uma postura crítica e, tampouco a visão global que eles devem ter sobre o ser humano que assistem. Por outro lado, acreditam que tanto o sistema de saúde quanto o empresariado do setor, objetivam um mais rápido atendimento, e de preferência, de mais baixo custo possível, evidentemente almejando a obtenção de lucro.

RODRIGUES \& LINS (1990) corroboram esta situação, comentando que existe uma crise envolvendo a enfermagem brasileira, trazendo os enfermeiros à uma realidade social, onde estão aprendendo a lidar com o não, com os limites e as frustrações. Os profissionais encontram-se limitados pela divisão de trabalho, com o surgimento de outros profissionais na área da saúde e pela falta de autonomia, consolidada nos serviços, pelos interesses dos grupos dominantes. Como o lucro consiste a essência do sistema capitalista, adotado no país, o enfermeiro não é visto ou entendido de forma holística e isto favorece com que ele mesmo transfira o modelo à sí imposto, quando divide tarefas entre os demais elementos da equipe de enfermagem, distribuindo-os em funções especializadas e, conseqüentemente, fragmentando a assistência. Com isto, acaba experimentando insatisfação, ansiedade, desestímulo, acomodação, atuação profissional frustada e sem esperança no futuro da profissão.

Diante do exposto, as dificuldades encontradas pelos docentes de Enfermagem Médica, em relação à insatisfação apresentada pelos graduandos de enfermagem, buscando se manter em um modelo tradicional de enfermagem, torna-se compreensível. Este entendimento é reforçado por SHOEMAKER (1989), para quem o enfermeiro não é preparado para realizar atribuições diagnósticas. As disciplinas iniciais de seu currículo de graduação deveriam introduzir o conteúdo de metodologia da assistência, a fim de possibilitar maior desenvolvimento deste processo, situação esta ainda não vivenciada com tranqüilidade, acredita-se, em várias escolas de enfermagem brasileiras.

No entanto, a persistência dos professores sobre a necessidade de alertarem os alunos sobre esse outro paradigma, continuou existindo. Muitas vezes as ocorrências vivenciadas com os pacientes, nos campos de realização de atividades práticas, não eram contempladas em salas de aulas, onde o conteúdo, pela questão formal de carga horária, não era possível de ser ministrado. No entanto, nos estágios, antes de elaborarem $\mathrm{DE}$, efetuarem prescrições e finalmente conseguirem realizar alguma intervenção de enfermagem, os alunos eram orientados a se instrumentalizarem cientificamente sobre aquele determinado conteúdo, desenvolvendo as habilidades de análise e síntese, no seu julgamento clínico.

CARVALHO et al. (1996), em estudo sobre DE, apontaram que os estudantes de anos anteriores, cursando a disciplina em questão, no nível educacional em que se encontravam, enfrentavam problemas de falta de conhecimento sobre fisiopatologia. Este fato contribuía para a limitada perspicácia em identificar dados de relevância, para conseguirem, finalmente, elaborarem os DE. A utilização de livros de fisiopatologias, de metodologias de assistência e a leitura de artigos 
científicos nos campos de estágio para a concretização desta instrumentalização, tornou-se então situação rotineira, para alunos e professores.

À todo este contexto, acrescia-se o fato de fazer parte, dentro do conteúdo da disciplina, aspectos específicos relativos à Enfermagem Neurológica, o que pressupõe, evidentemente, a realização das atividades práticas em clínicas de internação de pacientes com alterações neurológicas para alcançar o reforço desta temática. Estes enfermos costumavam e ainda tendem a provocar receios nos alunos, pelo fato, às vezes, de não terem capacidade de falar, mover-se, raciocinar, apresentarem enfermidades sem causas conhecidas, ou permanecerem com seqüelas físicas e/ou psicológicas/ emocionais. À este respeito, SMELTZER \& BARE (1994 a) comentam que por vezes, o doente neurológico experimenta alterações corporais, comprometimentos cognitivos, de visão ou fala e diminuição da auto-estima. Complementa SCHMIDT (1979) que, necessitando os neurônios de um abastecimento constante de oxigênio, interrupções da circulação cerebral por cerca de 8 a 10 segundos provocam pêrdas de consciência; após 8 a 10 minutos, a lesão cerebral provocada é irreversível, levando a pessoa a apresentar seqüelas, caso sobreviva.

Como uma lesão encefálica dificilmente consegue ser integralmente restaurada, surge o desenvolvimento de paralisias, comas, dores crônicas e distúrbios comportamentais, entre outros problemas de saúde. Assim aconteciam e ainda ocorrem dificuldades para a realização das primeiras abordagens realizadas pelos estudantes, que demonstram receios e aparentes incapacidades, em suas iniciações clínicas com estes pacientes. Era e ainda é comum ouvir-se questionamentos, do tipo: "...mas como vou realizar coleta de dados neste paciente, se ele está em coma? ....Ou não fala?...Ou não compreende o que pergunto?...".

Afirma PORTO (1982) que a doença altera a personalidade das pessoas e chega-lhes a determinar regressão, à níveis infantis de dependência, com pêrda de segurança e desenvolvimento de fantasias, que têm por objetivo inconsciente fugir da realidade. Existe uma gama de comportamentos apresentados pelos pacientes que é muito variada e os estudantes então, vão aprendendo a reconhecê-los com a experiência adquirida com o decorrer dos anos e com a incorporação de novos conhecimentos.

Necessitam então adquirir a habilidade de uma melhor comunicação. A este respeito, STEFANELLI (1990) recomenda que é necessário desenvolver a comunicação terapêutica, pois é só através desta que é possível realizar uma abordagem adequada do homem, em todas as suas dimensões. A mesma, portanto, deve ser tratada com maior cuidado pelos docentes das escolas de enfermagem, pelas enfermeiras do campo e também pelos alunos.

Obviamente, com o reforço deste conteúdo ministrado em salas de aula e com a orientação recebida dos professores, nos campos de atividades práticas, as impressões iniciais de dificuldades, manifestadas pelos alunos, na maioria das vezes, costumam ser superadas, com o prosseguimento das atividades práticas. Acreditase, no entanto, serem dificultadoras para a utilização do Processo de Enfermagem em todas as suas fases, incluindo-se a de efetivas intervenções e avaliações, no período de tempo estabelecido.

A presente investigação teve então como objetivos: 1. identificar as categorias diagnósticas, atribuidas pelos graduandos de enfermagem, aos pacientes com alterações neurológicas e 2. identificar os respectivos padrões de respostas humanas, a partir dos Diagnósticos de Enfermagem elaborados pelos alunos, a tais pacientes.

\section{METODOLOGIA}

O estudo realizou-se na cidade de Ribeirão Preto, Estado de São Paulo. Todos os estudantes do $5^{\circ}$ semestre do Curso de Graduação em Enfermagem da EERP-USP, que cursaram a Disciplina Enfermagem Médica, no ano de 1996, receberam conteúdos teóricos específicos sobre Assistência de Enfermagem a Pacientes com Alterações Cardio-respiratórias, Neurológicas, Renais, Hematológicas, Gastrointestinais, Endocrinológicas, além de conteúdos referentes as etapas da metodologia de assistência, incluindo-se os DE, segundo a Taxonomia 1 da NANDA (1994).

Para reforço dos conteúdos de Enfermagem Neurológica, além de livros gerais que continham informações sobre a fisiopatologia e o exame físico a ser realizado, em casos de doenças neurológicas, tais como os de PLUM \& POSNER (1977); SANVITO (1978); SCHMIDT (1979); PORTO (1982); entre outros, foram orientados a ler os conteúdos existentes, particularmente os que se relacionavam às atribuições diagnósticas, do livro-texto de SMELTZER \& BARE (1994 a,b), ou em edições mais recentes que encontrassem na biblioteca da Universidade. Estas orientações não foram averiguadas, de sorte que não se conheceu, com certeza, quais alunos cumpriram esta tarefa complementar de leitura.

Após a ministração desta carga horária teórica, foram subdivididos em grupos, para a realização das atividades práticas, nas clínicas de internação de um dos hospitais universitários, utilizado para este fim, pela EERP-USP. Foi-lhes entregue um roteiro, para facilitar a obtenção de dados, nas fases de entrevista e exame físico dos pacientes, construído de acordo com o modelo 
eclético citado por GRIFFITH KENNY \& CHRISTENSEN (1986). Onze alunos foram escalados para prestarem assistência de enfermagem à dez indivíduos internados com alterações neurológicas, cujos Diagnósticos Médicos, muitas vezes múltiplos, variaram entre Epilepsias, Demência, Doença de Creutzfeldt-Jacob, Lesões de Medula, Mielopatias, Tumores Cerebrais, Sífilis do Sistema Nervoso Central, Hidrocefalia, Esclerose Múltipla, Coma e Acidentes Vasculares Cerebrais, entre outros. Alguns destes conteúdos com DE atribuídos, foram abordados nas aulas teóricas, outros não. Os estudantes receberam então a incumbência de desenvolver, em duas semanas planejadas para o respectivo estágio, a assistência de enfermagem adotando a metodologia descrita anteriormente. Os resultados desta tarefa, encontram-se descritos a seguir.

\section{RESULTADOS E DISCUSSÃO}

Em relação ao primeiro objetivo (identificar as categorias diagnósticas, atribuídas pelos graduandos de enfermagem, aos pacientes com alterações neurológicas) observou-se que os estudantes elaboraram $28 \mathrm{DE}$ aos 10 pacientes assistidos. As informações referentes a distribuição dos DE, em cada um dos padrões encontrados no presente estudo, encontram-se nos gráficos que se seguem.

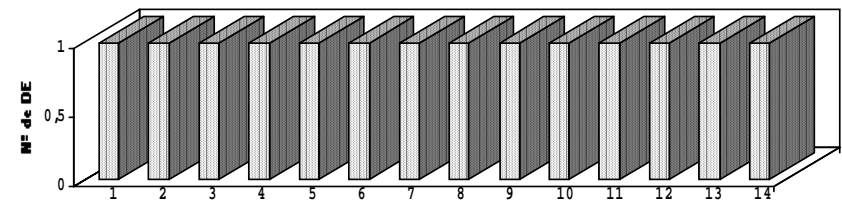

Gráfico 1 - Distribuição de Diagnósticos de Enfermagem (DE) segundo a Taxonomia 1 da NANDA, em pacientes com alterações neurológicas, segundo os Padrões de Respostas Humanas TROCAR

\section{TIPOS DE DIAGNÓSTICOS}

\begin{tabular}{|c|c|}
\hline 01 Eliminação Urinária Alterada & 02 Constipação \\
\hline $\begin{array}{l}03 \text { Integridade Tissular } \\
\text { Prejudicada }\end{array}$ & 04 Incontinência Intestinal \\
\hline 05 Proteção Alterada & 06 Incontinência Reflexa \\
\hline 07 Padrão Respiratório Ineficaz & 08 Potencial para Trauma \\
\hline $\begin{array}{l}09 \text { Alteração Nutricional para } \\
\text { menos }\end{array}$ & 10 Deglutição Prejudicada \\
\hline 11 Potencial para Aspiração & 12 Nutrição Alterada \\
\hline $\begin{array}{l}\text { 13 Potencial para Integridade da } \\
\text { Pele Prejudicada }\end{array}$ & $\begin{array}{l}14 \text { Déficit de Volume de } \\
\text { Liquido }\end{array}$ \\
\hline
\end{tabular}

Em relação aos DE deste padrão, observa-se que cada um significou $3,57 \%$ em relação ao número total de atribuições diagnósticas.

Estes $14 \mathrm{DE}$ atribuídos, no que se refere aos 28 elaborados, representam $50 \%$ deste total. Acredita-se que os mesmos são compreensíveis de serem encontrados em pacientes com alterações neurológicas, tais como as citadas anteriormente, ou seja, que não deambulam, encontram-se restritos aos leitos, com presença de escaras de decúbito, emagrecidos, com incapacidades para falar, alimentar-se sozinhos, com presença de sondas nasogástrica e/ou naso-enteral, traqueostomias, entre outras situações, Outrossim, supõe-se que se o período destinado a este estágio específico fosse maior, considerando-se as barreiras iniciais apresentadas pelos alunos, na aceitação dos $\mathrm{DE}$ e em relação às abordagens aos pacientes com problemas neurológicos, outros diagnósticos, inseridos neste e em outros padrões, certamente seriam atribuídos.

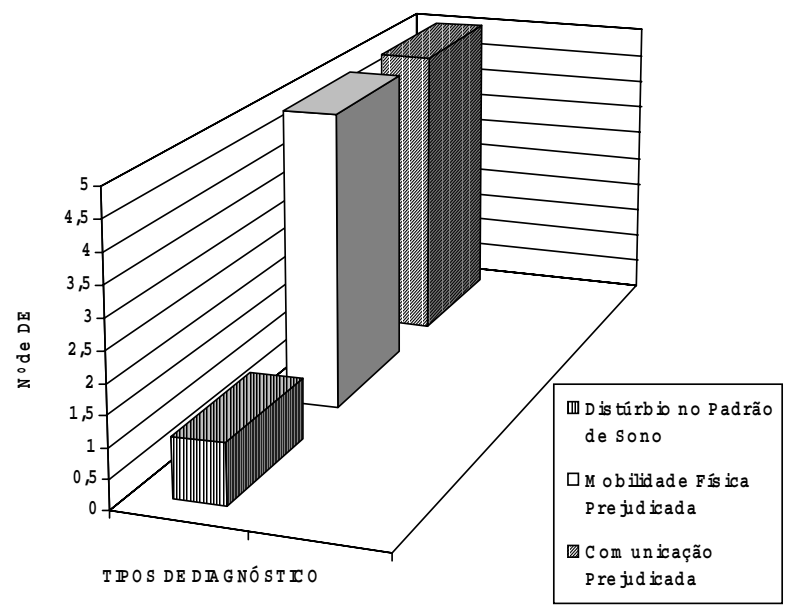

Gráfico 2 - Distribuição de Diagnósticos de Enfermagem (DE) segundo a Taxonomia 1 da NANDA, em pacientes com alterações neurológicas, segundo os Padrões de Respostas Humanas COMUNICAR E MOVER

Em relação ao Padrão Comunicar, foram encontrados 5 (cinco) DE de Comunicação Prejudicada, o que representou $18 \%$ do total de 28 DE elaborados. Este é um dos diagnósticos que se espera encontrar em pacientes com distúrbios neurológicos.

Muitas vezes com a função cognitiva comprometida, com alterações respiratórias severas, utilizando-se de cânulas orotraqueais ou de traqueostomia, com lesões que provocam paralisias da musculatura responsável pela fonação ou em estado de coma, decorrente das mais variadas causas (SANVITO, 1978), torna-se difícil a comunicação com os mesmos. Conforme atestam SMELTZER \& BARE (1994 a), podem acontecer entre estes enfermos distúrbios de auto- 
estima relacionados à perda da capacidade de comunicação, déficits de comunicação relacionados às lesões cerebrais, incluindo-se comprometimento das relações familiares, em virtude deste problema. A enfermeira muito tem a realizar nos aspectos relativos à melhoria da comunicação com estes pacientes.

Quanto ao Padrão Mover, também apresentado no Gráfico 2, os alunos conseguiram atribuir 5 (cinco) DE de Mobilidade Física Prejudicada que representam $17,85 \%$ em relação aos 28 DE elaborados e 1 (um) de Distúrbio no Padrão do Sono $3,57 \%$ em relação a este total. BACHION et al. (1995) igualmente encontraram DE relatados neste padrão, no estudo que realizaram em indivíduos com alterações cardíacas. Como era de se esperar, no entanto, os pacientes cardíacos apresentaram estes tipos de diagnósticos em freqüências menores que as encontradas nos enfermos com distúrbios neurológicos.

As disfunções relativas à falta de movimentação são comuns de ocorrerem nestes últimos. Perdas de movimentos no hemicorpo, paraplegias ou quadriplegias, ou imobilidades generalizadas em decorrência de estados comatosos, são costumeiramente encontradas nestes indivíduos, provocando ainda outras seqüelas, como escaras de decúbito, atrofias nos dimídios que perderam a capacidade de mover-se, alterações de posturas, deformidades físicas, entre outras. Os livros textos básicos de patologias neurológicas, citados anteriormente, tais como os de PLUM \& POSNER (1977); SANVITO (1978); SCHMIDT (1979); PORTO (1982); entre outros, reforçam tais problemas nestes pacientes.

O Distúrbio no Padrão do Sono foi encontrado em um paciente jovem, bem apessoado, em situação econômica e de conhecimento aparentemente superior aos demais enfermos que se encontravam internados no hospital universitário. Havia sido hospitalizado para investigação diagnóstica, já que apresentava crises convulsivas esporádicas, sem causas aparentes. Demonstrando condicionamento físico, praticava esportes freqüentemente, antes da hospitalização; no entanto sentia dificuldades para dormir, em decorrência da mudança de ambiente e presença de outros pacientes no quarto, pleiteando permanecer sozinho em uma das enfermarias. Durante o estágio dos graduandos de enfermagem, evidenciou-se através de realização de exame especializado, que possuía um certo tipo de tumoração encefálica, com necessidade premente de intervenção cirúrgica e com prognóstico incerto quanto à sua cura. A partir daí, tornou-se extremamente nervoso, aumentando a sua dificuldade para dormir, mesmo com a mudança de enfermaria, o que justificou o DE elaborado pelo estudante que $\mathrm{o}$ assistia.

Em relação aos Padrões Sentir, Escolher e Perceber, encontram-se visualmente apresentados à seguir.

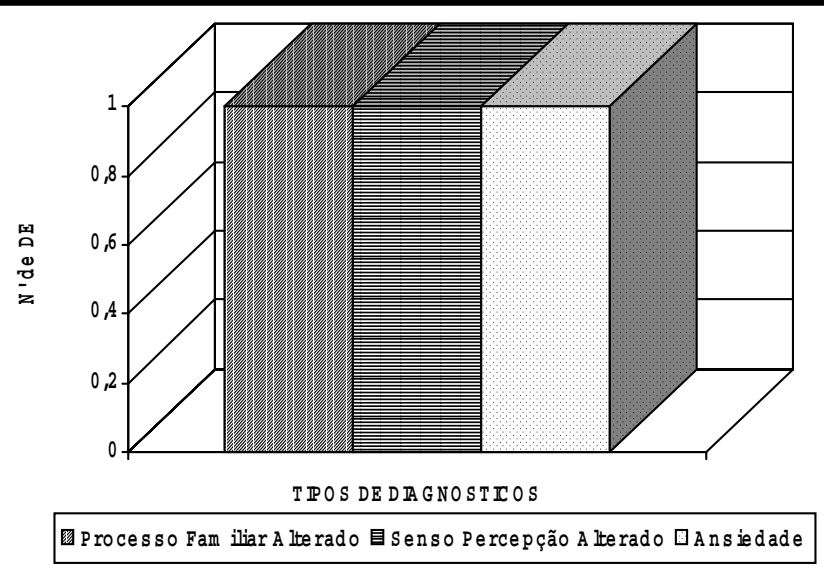

Gráfico 3 - Distribuição de Diagnósticos de Enfermagem (DE) segundo a Taxonomia $1 \mathrm{da}$ NANDA, em pacientes com alterações neurológicas, segundo os Padrões de Respostas Humanas SENTIR, ESCOLHER A PERCEBER

Observa-se que dentro do padrão Sentir, foi atribuído o DE de Ansiedade, em freqüência de 3,57\% em relação ao total de diagnósticos atribuídos; inserido no Padrão Perceber o DE de Senso Percepção Alterada e no Padrão Escolher, o diagnóstico de Processo Familiar Alterado, todos encontrados com a mesma percentagem. São compreensíveis de serem encontrados em pacientes com alterações neurológicas.

Ansiedade foi DE também encontrado por BACHION et al. (1995), em pacientes com alterações cardíacas, só que em percentagem inferior ao do atual estudo. Esta manifestação diagnóstica foi atribuída ao paciente com tumoração cerebral relatado anteriormente, que se sentia ameaçado frente à perspectiva de cirurgia iminente, com grandes riscos de lhe provocar paralisias, e começou a apresentar episódios de impaciência, inquietação e expressão facial tensa, entre outras. Explica PORTO (1982) que o paciente ansioso apresenta manifestações psíquicas e somáticas que a acompanham, tais como inquietude, voz embargada, mãos frias, trêmulas e/ou sudoréticas, taquicardias, boca seca, entre outros problemas. SMELTZER \& BARE (1994 a) ainda acrescentam que o enfermeiro deve estar atento ao preparo emocional dos pacientes que vão ser submetidos à cirurgias intracranianas e, inclusive, caso seja possível, deve incluir informações que satisfaçam as expectativas pós-operatórias dos mesmos. Apoio e consideração aos enfermos e seus familiares devem ser ofertados, pois segundo as palavras destes autores, reconhece-se "a potencial gravidade de uma cirurgia intracraniana". Obviamente não é difícil compreender as manifestações de ansiedade presentes no paciente em questão, particularmente em se tratando de um indivíduo com certa diferenciação econômica e de conhecimento, em relação aos demais pacientes internados na mesma clínica.

O DE de Senso Percepção Alterada foi atribuído 
a um paciente epiléptico, de 16 anos, que se manifestava habitualmente aos alunos através de olhar fixo, parado, modificações abruptas de labilidade emocional, "ausências" do ambiente e lentidão de funções psíquicas, entre outras situações. SMELTZER \& BARE (1994 b) observam que problemas de ordens social, psicológica e comportamental, freqüentemente acompanham as Epilepsias, podendo até mesmo ser mais incapacitantes que as próprias convulsões. Este mesmo paciente apresentou o DE de Processo Familiar Alterado também em percentagem de 3,57, inserido no Padrão Escolher: era filho adotivo e manifestou sentimentos negativos, particularmente em relação ao pai, que segundo ele, o rejeitava.

Estes diagnósticos reforçam as considerações sobre o tratamento dados à crianças epilépticas, que costumam ser excluídas das atividades de escola e dos companheiros; segundo SMELTZER \& BARE (1994 b), estes problemas parecem ser aumentados na fase de adolescência, somando-se aos desafios dos encontros, inabilidades para executar algumas atividades e a presença do sentimento de "ser diferente". A tentativa do aluno de enfermagem em conversar sobre o assunto com os familiares, mostrou-se também infrutífera, com os mesmos contradizendo o sentimento experienciado pelo paciente. Os autores anteriormente citados reportamse ao que denominam "inequívoca rejeição" de paciente com tal patologia, por parte da família, porque esta já carrega uma grande carga, em decorrência dos variados problemas apresentados pelos pacientes. Acresce-se a isso, a inabilidade que pode ter acontecido com o estudante, na fase de obter informações complementares, dos familiares do enfermo em questão.

No que se refere ao alcance do segundo objetivo (identificar os respectivos padrões de respostas humanas, a partir dos Diagnósticos de Enfermagem elaborados pelos alunos, a tais pacientes) ao se verificar a distribuição dos DE de acordo com estes padrões, em cada um dos 10 pacientes assistidos, elaborou-se a Tabela 1 que se segue.

Tabela 1 - Distribuição de Diagnósticos de Enfermagem (DE) e de padrões de respostas humanas, segundo a Taxonomia 1 da NANDA em pacientes com alterações neurológicas

\begin{tabular}{|c|c|c|c|c|c|c|c|}
\hline $\mathrm{N}^{\circ}$ pacientes & Trocar & Mover & Comunicar & Perceber & Escolher & Sentir & $\mathrm{N}^{\circ}$ de $\mathrm{DE}$ \\
\hline 1 & 3 & 1 & 1 & & & & 5 \\
\hline 1 & 2 & 1 & 1 & & & & 4 \\
\hline 1 & 2 & & 1 & & & & 3 \\
\hline 1 & 1 & 1 & 1 & & & & 3 \\
\hline 1 & 2 & 1 & & & & & 5 \\
\hline 1 & 2 & 1 & & & & & 3 \\
\hline 1 & 1 & & 1 & & & & 2 \\
\hline 1 & & & & 1 & 1 & & 2 \\
\hline 1 & & 1 & & & & 1 & 2 \\
\hline 1 & 1 & & & & & & 1 \\
\hline Total 10 & 14 & 6 & 5 & 1 & 1 & 1 & 28 \\
\hline
\end{tabular}

Ao se tabular estas informações, observou-se uma variação de no mínimo 1 (um) ao máximo de 5 (cinco) DE para cada paciente, notando-se um valor médio de 2.8 DE. Este fato é importante, à medida em que reforça aos professores a questão de estarem alcançando o ideal do ensino de uma Enfermagem individualizada, personalizada, planejada para cada pessoa a ser assistida, como é o entendimento que possuem sobre o Processo de Enfermagem, com as atribuições diagnósticas e com a questão maior da modificação do paradigma da profissão Enfermagem. À medida em que alguns enfermos com Diagnósticos Médicos semelhantes, como os três (3) primeiros pacientes, mostrados na Tabela 1 , receberam no total, 12 DE distintos; os dois (2) pacientes que encontram-se em seguida receberam seis (6) DE e os outros dois (2) mais cinco (5) atribuições diagnósticas, considerou-se que a metodologia de assistência empregada havia cumprido a sua primeira "tarefa".

Parte-se então do princípio que o "antigo" enfermeiro, enquanto elemento "acrítico", "cumpridor de ordens e de tarefas" ordenadas por outros profissionais, até então tidos como os principais detentores do "saber", lamentando-se de sua vida profissional e não conseguindo revertê-la, possivelmente poderá deixar de existir, em anos vindouros, quando descobrir o seu potencial enquanto elemento com capacidade de adquirir 
conhecimentos, de diagnosticar, em busca deste novo "olhar para a Enfermagem", relacionado à qualidade do cuidado a ser prestado aos seus clientes, voltado à assistência especializada, não alienada, à consciência crítica, a um processo de participação profissional ativa. A distribuição gráfica dos DE segundo estes padrões, encontram-se a seguir.

A Tabela 1 pode ser sintetizada no gráfico a seguir.

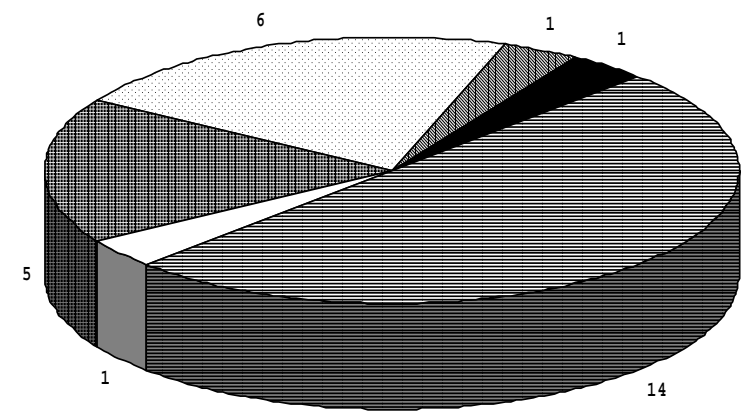

目 trocar $\square$ sentir 团 com un icar $\square \mathrm{m}$ over $\mathbb{\mathbb { N }}$ perceber $\mathbf{U}$ escolher

Gráfico 4 - Distribuição de padrões de respostas humanas, segundo a Taxonomia 1 da NANDA, em pacientes com alterações neurológicas

Como pode ser observado, a maior parte dos estudantes realizou atribuições diagnósticas referentes ao Padrão Trocar (50\%), seguidas dos Padrões Mover (21\%), Comunicar (18\%), do Padrão Sentir (4\%), do Escolher $(4 \%$,$) e do Perceber (4\%). À semelhança do estudo$ realizado por BACHION et al. (1995), ainda observa-se no alunado de Enfermagem Médica, tendência a valorizar, as alterações biológicas apresentadas pelos doentes que assistem, em detrimento das demais, o que fica comprovado pela maior facilidade em atribuir diagnósticos inseridos nos Padrões Trocar e Mover.

Pode ser que a predominância desta situação tenha ocorrido em decorrência da orientação dada para a fase de coleta de dados, abrangendo entrevista e exame físico, com roteiro previamente entregue aos estudantes. PORTO (1982) enfatiza que saber entrevistar pacientes constitui-se em um processo social, de interação entre o profissional e o enfermo. Há que se garantir um ambiente propício para isto, o que muitas vezes torna-se difícil em hospitais universitários; deve ocorrer uma predisposição do paciente em fornecer informações, o que também pode tornar-se uma condição dificultada, pelo acúmulo de estudantes e outros profissionais que entrevistam o mesmo indivíduo, várias vezes ao dia.

Além disso, complementa PORTO (1982) que a linguagem empregada para se obter a entrevista, em alguns momentos torna-se ininteligível para os entrevistados; os quadros de referências existentes, em geral, são distintos, tanto por parte do profissional, quanto do aluno, como do paciente. Existem diferentes culturas entre estas pessoas; a reação dos pacientes pode variar, ante os estímulos apresentados pelos entrevistadores e vice-versa. Estes, entre outros, podem ser elementos dificultadores, para se obter uma entrevista. $\mathrm{O}$ exame físico complementar à abordagem inicial possui um grande significado psicológico para os enfermos e adquirir habilidades suficientes para realizá-lo com adequacidade, demanda experiência, tempo, respeito mútuo, seriedade e segurança, aspectos estes que não estão prontos, mas vêm sendo adquiridos, de modo gradual, pelos estudantes, em seu processo de aprendizagem.

Acrescenta-se a este fato a situação usualmente encontrada nos pacientes com alterações neurológicas, já comentadas por SMELTZER \& BARE (1994a, b) particularmente os que apresentam seqüelas destas enfermidades. Acredita-se que "o que chama a atenção inicial do examinador", em geral, é a parte física, que no caso de indivíduo com patologia neurológica, muitas vezes, encontra-se lesada, observando-se presença de deformidades, cabeças alopécicas, órgãos do sentido alterados, marchas sem simetria, quedas ao solo, conversas desconexas, aparentando que as pessoas encontram-se, as vezes, com distúrbios psiquiátricos, entre outras situações.

Como em geral, as capacidades físicas dos pacientes são as inicialmente procuradas pelos estudantes, para darem início ao processo interacional com os enfermos, particularmente no ato de realização da primeira entrevista, é de se esperar que as valorizem, mais que as outras. Afirma STEFANELLI (1990) que fala-se muito sobre a importância do enfermeiro saber comunicar-se, sobre a necessidade que ele tem de desenvolver competência no relacionamento interpessoal, mas na prática, de um modo geral, isso não é observado. FERRAZ et al. (1995) acrescentam que os enfermeiros não conhecem as estratégias de comunicação terapêutica e os modos de comunicação não-terapêutica, apesar de terem tido este conteúdo em algum momento de sua formação acadêmica. Esta dificuldade inicia-se no Curso de Graduação e provavelmente agrava-se, caso os estudantes iniciem seus contactos com pacientes portadores de distúrbios relacionados à comunicação e, possivelmente, prossegue durante toda a vida profissional, se não for desestimulada pelos professores, através do estabelecimento de uma relação de ajuda aos alunos.

Além disso aprender a comunicar-se efetivamente com os doentes, significa, em instância maior, iniciar os primeiros passos para tornar-se um agente ativo da ação de realizar Enfermagem, assumindo um compromisso para com a profissão e uma postura de 
domínio da situação e não apenas de mero espectador da mesma.

SMELTZER \& BARE (1994 a) citam que a área de Enfermagem Neurológica possibilita a realização profissional ao enfermeiro, pois proporciona a oportunidade de reabilitar funcionalmente os enfermos, que encontram-se com perdas decorrentes de fraquezas, imobilidades, comprometimentos de reflexos posturais, dores articulares entre outros. Ou seja, é particularmente a capacidade física que aparenta ter a maior alteração e, praticando a reabilitação, o profissional de Enfermagem, consegue melhorar a qualidade de vida destas pessoas.

A tendência de estimação dos aspectos estritamente biológicos, no entanto, foi reduzida em relação ao levantamento efetuado com alunos do mesmo semestre letivo, em 1995, por BACHION et al.(1995), pois mesmo apesar de somente 11 alunos, 10 pacientes e 28 atribuições diagnósticas relatadas, estarem sendo considerados no atual estudo, observou-se que padrões de respostas humanas, anteriormente não tão claramente "percebidos" pelos estudantes, passaram a ser contemplados por eles, como ocorreu, por exemplo, com os Padrões Comunicar, Perceber e Escolher.

Pode-se supor então que os docentes da disciplina em questão, em esforço conjunto, incrementaram o ensino sobre estas outras abordagens além da predominantemente biológica, mesmo em se tratando de enfermos com problemas neurológicos, na tentativa cada vez maior de procurarem levar à compreensão dos estudantes que o homem a ser assistido deve ser contemplado de forma integral, holística, por ser criatura indivisível e não apenas portador de um corpo físico e visível. Acreditam, como explica BACHION et al. (1995), que para acontecer este tipo de abordagem global, é necessário que ocorra uma busca de dados, abrangendo diferentes e mais "profundos" aspectos, para que os demais padrões sejam encontrados. A abordagem eclética adotada para construir o instrumento de coleta de dados, favorece a aquisição de dados de todos os padrões.

A intencionalidade dos professores em desenvolver nestes alunos, habilidades de análise e síntese no julgamento clínico, parece então estar resultando em experiência positiva, formando-se por referência o perfil de Padrões de Respostas Humanas encontrados neste estudo.

\section{CONCLUSÕES E CONSIDERAÇÕES}

- 10 pacientes com alterações neurológicas, internados em um hospital universitário da cidade de Ribeirão Preto, foram assistidos por 11 alunos do $5^{\circ}$ semestre do Curso de Graduação em Enfermagem, cursando a Disciplina
Enfermagem Médica, na EERP-USP e receberam vinte e oito diferentes atribuições diagnósticas, segundo a Taxonomia 1 da NANDA (1994);

- estes DE encontravam-se inseridos, predominantemente, no Padrão de Resposta Humana Trocar (50\% das atribuições diagnósticas), seguidos do Padrão Mover (21\%), Comunicar (18\%), Escolher (4\%), Perceber (4\%) e Sentir (4\%);

- os DE do Padrão Trocar foram, na frequência de 3,57\% cada um: Eliminação Urinária Alterada, Constipação, Integridade Tissular Prejudicada, Incontinência Intestinal, Proteção Alterada, Incontinência Reflexa, Padrão Respiratório Ineficaz, Potencial para Trauma, Alteração Nutricional para Menos, Deglutição Prejudicada, Potencial para Aspiração, Nutrição Alterada, Potencial para Integridade da Pele Prejudicada e Déficit de Volume de Líquido;

- os DE do Padrão Mover foram 5 (cinco) de Mobilidade Física Prejudicada que representam 17,85\% em relação aos 28 DE elaborados e 1 (um) de Distúrbio no Padrão do Sono, que significa 3,57\% em relação a este total; em relação ao Padrão Comunicar, foram encontrados 5 (cinco) DE de Comunicação Prejudicada, o que representou $18 \%$ do total de 28 DE elaborados;

- quanto ao Padrão Sentir, foi atribuído o DE de Ansiedade, em freqüência de $3,57 \%$ em relação ao total de atribuições; inserido no Padrão Perceber encontrouse o DE de Senso Percepção Alterada e no Padrão Escolher, o de Processo Familiar Alterado, todos encontrados com a mesma percentagem;

- observou-se ainda predominância, por parte dos alunos de valorização dos DE que tratam de alterações biológicas, embora em menor percentagem ao estudo anterior realizado, com estudantes do mesmo semestre, por BACHION et al. (1995);

- é esperado de se encontrar, em pacientes com alterações neurológicas, predominância de maior número de alterações físicas, que de outra natureza, conforme constata a literatura, o que justifica então os DE atribuídos à estes enfermos;

- houve atribuição de DE de Padrões que não haviam sido contemplados anteriormente, no estudo de BACHION et al. (1995), tais como os dos Padrões Comunicar e Escolher, significando, provavelmente, que os professores, em esforço conjunto, conseguiram alertar os estudantes à observação das outras características dos pacientes, além das predominantemente biológicas; - o tempo para a concretização das atividades práticas, acrescido à inexperiência por parte dos estudantes em efetuar as fases de entrevista e exame físico, além de outros fatores como ausência de local adequado para a realização desta etapa do Processo de Enfermagem, interação ainda não inteiramente adequada entre alunos 
e pacientes neurológicos, podem ter interferido na atribuição de outros DE, inseridos em outros Padrões de Respostas Humanas;

- não foram contemplados DE dos padrões Conhecer, Relacionar e Valorizar, o que talvez possa ser minimizado em anos posteriores, com a maior ênfase destes outros aspectos na disciplina Enfermagem Médica, bem como a compreensão de que podem ser menos fáceis de serem percebidos, em pacientes com alterações neurológicas e com dificuldades de comunicação;

- a intencionalidade dos docentes da Disciplina Enfermagem Médica em desenvolver habilidades de análise e síntese no julgamento clínico, em alunos de graduação em Enfermagem, tem-se mostrado como uma experiência positiva, tomando-se por referência o perfil de padrões de respostas humanas atribuídas neste relato.

NURSING DIAGNOSES ATTRIBUTED BY NURSING UNDERGRADUATE STUDENTS TO IN PATIENTS (ADMITTED IN HOSPITAL) WITH NEUROLOGIC ALTERATIONS

Diagnostic categories and patterns of human responses were identified in neurologic patients, assisted by nursing undergraduate students who attributed to them nursing diagnoses, according to NANDA's Taxinomy I. The majority of attributed diagnoses refer to the patterns Exchange, Move and Communicate and the minority to the patterns Choose, Receive and Feel. The non attribution of other diagnoses probably occurred due to the lack of emphasis given to them and to students' inexperience. Faculty determinations to develop in the students skills of analysis and synthesis confirm this positive experience.

KEY WORDS: attribution of nursing diagnoses to neurologic patients; teaching of nursing diagnoses; nursing process

\section{DIAGNÓSTICOS DE ENFERMERÍA: ATRIBUCIÓN HECHA POR ESTUDIANTES DE PRE- GRADO EN ENFERMERÍA A PACIENTES INGRESADOS CON ALTERACIONES NEUROLÓGICAS}

Fueron identificadas categorías diagnósticas y padrones de respuestas humanas en pacientes neurológicos, asistidos por alumnos de pre-grado, que les atribuyeron diagnósticos de enfermería según la Taxonomía I de la NANDA. La mayoría de los diagnósticos atribuidos se refieren a los patrones Cambia, Mover y Comunicar y la minoría a los patrones Escoger, Recibir y Sentir. La no atribución de otros diagnósticos ocurrió provablemente por el menor énfasis dado a los mismos y la inexperiencia de los alumnos; la intencionalidad de los docentes en desarrollar en los estudiantes habilidades de análisis y síntesis, se ha mostrado una experiencia positiva.

TÉRMINOS CLAVES: atribución de diagnósticos de enfermería a pacientes neurológicos, enseñanza de diagnósticos de enfermería, proceso de enfermería

\section{REFERÊNCIAS BIBLIOGRÁFICAS}

01. BACHION, M.M.; ROBAZZI, M.L.C.C.; CARVALHO, E.C.; VEIGA, E.V. Clientela com alterações cardíacas: perfil diagnóstico elaborado por alunos de enfermagem. Rev.latino-am. Enfermagem, Ribeirão Preto, v.3, n. 2, p. 8392, jul. 1995.

02. CARVALHO, E.C.; BACHION, M.M.; FERRAZ, A.E.P.; VEIGA, E.V.; RUFFINO, M.C.; ROBAZZI, M.L.C.C. O processo de diagnosticar e o seu ensino. São Paulo, Rev.Esc.Enfermagem USP, v.30, n. 1, p. 33-43, abr. 1996.

03. FARIAS, J.N. et al. Diagnósticos de enfermagem: uma abordagem conceitual e prática. João Pessoa: Santa Marta, 1990.
04. FERRAZ, A.F.; ALVES, M.A.; PEIXOTO, M.R.B. Comunicação terapêutica na prática profissional e social do enfermeiro. Rev.Gaúch. Enfermagem, Porto Alegre, v. 16, n. 1/2, p.2129, 1995.

05. GRIFFITH-KENNEY, J.W.; CHRISTENSEN, P.J. Nursing process: application of theories, frameworks and models: a multifocal approach to individuals, families and communites. 2. ed. St. Louis: Mosby, 1986.

06. NANDA-NURSING DIAGNOSIS. Definitions \& classification. North American Nursing Diagnosis Association, 1994. 114 p.

07. PLUM, F.; POSNER, J.B. Diagnóstico de estupor e coma. 2. ed. Rio de Janeiro: Guanabara Koogan, 1977. $360 \mathrm{p}$.

08. PORTO, C.C. Iniciação ao exame clínico. In: PORTO, C.C. Exame clínico. Rio de Janeiro: Guanabara Koogan, 1982. p. 1-16. 
09. RODRIGUES, E.M.D.; LINS, L.C.S. Enfermagem no Nordeste - momento e tendências. Enfermagem Científica, Rio de Janeiro, n. 1, p. 28-31, 1990.

10. SANVITO, W.L. Os comas na prática médica. São Paulo: Manole, 1978. 545 p.

11. SCHMIDT, R.F. (org). Neurofisiologia. São Paulo: EPU/SPRINGER/EDUSP, 1979. 372 p.

12. SHOEMAKER, J.K. Nursing diagnosis in graduate curricula. J.Prof.Nurs., v.5, n. 3. p.140-43, 1989.
13. SMELTZER, S.C.; BARE, B.G. Brunner/Suddarth: tratado de enfermagem médico-cirúrgica. 7. ed. Rio de Janeiro: Guanabara Koogan, 1994 a. Cap. 56, p. 1395-1440: Tratamento de pacientes com distúrbios neurológicos.

14. SMELTZER, S.C.; BARE, B.G. Brunner/Suddarth: tratado de enfermagem médico-cirúrgica. 7. ed. Rio de Janeiro: Guanabara Koogan, 1994 b. Cap. 57, p. 1441-1505: Tratamento de pacientes com doenças neurológicas.

15. STEFANELLI, M. C. Comunicação terapêutica enfermeira-paciente avaliação de ensino. Enfermagem Científica, Rio de Janeiro, n. 1, p. 4-10, 1990. 